\title{
HIGH-PRECISION DISTANCE MEASUREMENT WITH AN UNMANNED, MOVING TARGET
}

\author{
by \\ H. Ito \\ (Alfred Wegener Institute für Polarforschung, D-2850 Bremerhaven, Federal Republic of Germany)
}

and

\section{K. Schroff and Hans-jörg Frei}

(Geographisches Institut, ETH Zürich, CH-8057 Zürich, Switzerland)

\begin{abstract}
A device was developed, which rotates a marker for the distance measurement in the desired direction, without an operator at the spot. This is also possible with a moving object, on which the marker is set up. The device, together with adequate distance-measuring instruments, yields a precise distance measurement by a single observer at a single station. Examples of measurements using it during sea-ice studies in the Greenland Sea are described. It is shown that the application of the instrument provides a satisfactory basis for precise glacier surveying with limited personnel, cost, and time.
\end{abstract}

\section{INTRODUCTION}

Glacier movement can of ten be determined only by conventional surveying on the ground, when high accuracy is required, while indirect methods, e.g. through satellites, may gain future importance. The ground survey consists of angle and distance measurements. The angle measurement raises fewer problems and is not discussed in this article. The distance is measured by the principle described below: a signal (e.g. laser, visible light, infra-red, micro-wave, sound) is sent from a marker to the observing station. The signal may be generated at the observing station, at the marker, or somewhere else. Correspondingly, the marker assumes an adequate form: reflector, transponder, transmitter, natural glacier surface, etc. The signal received at the observing station is used to compute the distance from the marker to the station, using, e.g., travel time, phase difference, intensity etc. As each type of distance measuring instrument has its merits and drawbacks, the instruments can be classified in various ways. For the current purpose, a classification is made based on a single aspect, namely whether the signal from the marker is or is not beamed toward the observing station. The former system gives generally better accuracy than the latter, due to its stronger signal at the observing station, and is used when the precise determination of the glacier motion, hence of the distance, is required. An operational problem in the application of this type of distance measuring instrument is discussed below.

The marker constructed for the purpose must be oriented so that it sends the beamed signal in the right direction. This is relatively simple to achieve for a single measurement. However, when a moving object, such as a glacier, is measured repeatedly, the orientation of the marker has to be re-adjusted for each reading, as the motion of the object rotates the marker relative to the observing station. Consequently, an operator is required to stay at each marker. This is inconvenient, if not impossible (danger, expensive, time consuming etc.), when many markers are measured repeatedly, which is the case for the glacier survey. This article suggests a way to solve this problem. There is an additional consideration. In certain circumstances, some risk of loss of, or damage to, the marker through the glacier motion must be allowed for, especially when the marker is unmanned. The marker should thus be regarded as semi-disposable. In fact, fully-automatic, distance-measuring instruments, e.g. Polarfix, available on the market, solve the problem described above, if costs are not considered.

A device is developed, which brings the marker into the right orientation without an operator at the spot, whatever motion the glacier assumes, so that sufficiently strong signals are received at the observing station. The production cost of the device is kept so low that occasional loss of the marker with the device does not cause a serious problem. With this device and a distance-measuring instrument, the glacier movement can be surveyed precisely by a single observer at a single station.

\section{INSTRUMENTS}

For test purposes, three different units, types A, B, and $\mathrm{C}$, were developed. For the experiment, a WILD Distomat DI4L was chosen as the distance-measuring instrument, with reflectors as markers. The word "reflector" is used below, instead of the general expression, "marker", as used in the preceding section. One of the three types of device is inserted between the reflector and a tripod. It rotates the reflector into the desired orientation. The mechanism for this is different for each type.

Type A: The device consists of a motor with a train of gears and rotates the reflector with a constant speed of 70 degree $\mathrm{min}^{-1}$, relative to the tripod. The power is supplied by a battery. The simple construction keeps both the production cost and frequency of failure low. Another merit is the minimum chance of freezing up, as it is in continuous motion. The measurement can, however, only be realized at a fixed time, i.e. when the reflector faces the observer by itself. The waiting time, until the reflector is rotated to the right orientation, can be as much as five minutes for this particular construction.

Type B: This type of mechanism rotates the reflector much faster than Type A and is equipped with a switch, which is remotely controlled by the distant observer. By means of a commercially-available control board for model aircraft, the observer can rotate the reflector either clockwise, or counter-clockwise, or stop it. While this type involves additional complication both in construction and operation, it makes the measurement possible at any time and the measurement can be continued as long as desired by keeping the reflector always facing the observer during the time length required.

Type C: The third type also has a remote-control mechanism, but the command is given in terms of the angle of rotation. The observer reads the amount of the signal he transmits on the scale of the slider (usually used to change the wing angle of a model aircraft) and converts it to the rotation angle of the reflector. By this procedure, the angle the glacier surface has rotated since the last measurement is obtained, unless the rotation exceeds $360^{\circ}$. 


\section{EXPERIMENTS}

There has been no opportunity yet to test the devices on a glacier, but they were used on the pack ice. Since the conditions were even harder (i.e. rapid motion) than those expected on a glacier, an application to a glacier survey should also be feasible.

The experiment was carried out in July 1983 as a part of MIZEX (Marginal Ice Zone Experiment) in the Greenland Sea, $79^{\prime} 40^{\prime} \mathrm{N}, 2^{\circ} \mathrm{E}$ (inset, Fig.1). The area was occupied by ice floes of various sizes. Most of them were multi-year ice with thickness of 3 to $5 \mathrm{~m}$. While the field was moving as a whole at a speed of approximately $0.2 \mathrm{~m}$ $\mathrm{s}^{-1}$, the relative motion within the area produced a deformation. To measure the deformation of the sea surface, markers were distributed in the area, and repeated positioning of these markers with high accuracy was required. The device discussed in this article had been developed primarily for this purpose. Results of the pack ice deformation study are given elsewhere (Ito, to be published).

One main station, $\mathrm{M}$, and three auxiliary stations, 1, 2 , and 3 , were established at the corners of a square, with a side length of $2 \mathrm{~km}$, on the pack ice field. The main station was equipped with a theodolite, Wild T2, a Wild Distomat DI4L and a transmitter for the remote control system. Each auxiliary station was equipped with one of the three types of the device, placed on a tripod and carrying a reflector with three prisms on top. The distances (and angles) from the main station to the auxiliary stations were measured repeatedly every 30 minutes for eight hours.

Fig.1 shows the location of the four stations at the beginning (left in the figure) and at the end of the

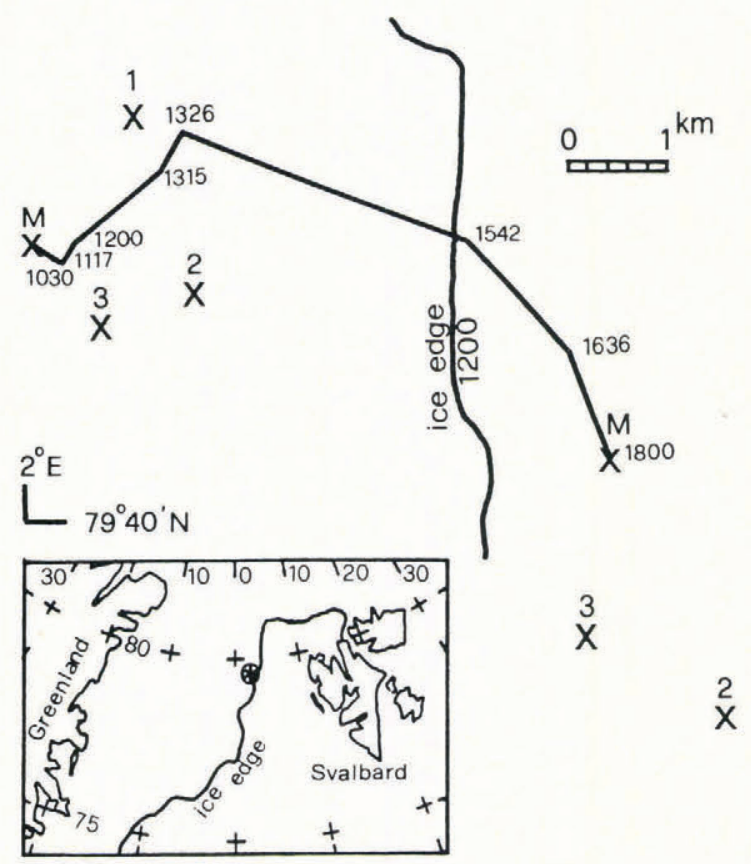

Fig.1. Position of the stations (M, 1, 2, and 3) at 1030 GMT and 1800 GMT, 11 July 1983. The time along the path of the main station (M) is in GMT of the day.

experiment (right). The path of the main station and the ice edge at $1200 \mathrm{Z}$ are also plotted in the figure. As the ice edge also moved, none of the stations crossed the ice edge during the experiment. The geographical position was determined by the ship's navigation system, while the relative position between stations was surveyed precisely, using the method described in this article. Hence, the accuracy is different for the absolute and relative positions by as much as three orders of magnitude. The ice-edge location, determined by visual observation, has even less accuracy. Fig.1 shows a combination of these three measurements of different precision and therefore presents solely an overview.

Fig.2 shows the rotation of the floe, on which the main station was located. It was recorded every 15 minutes

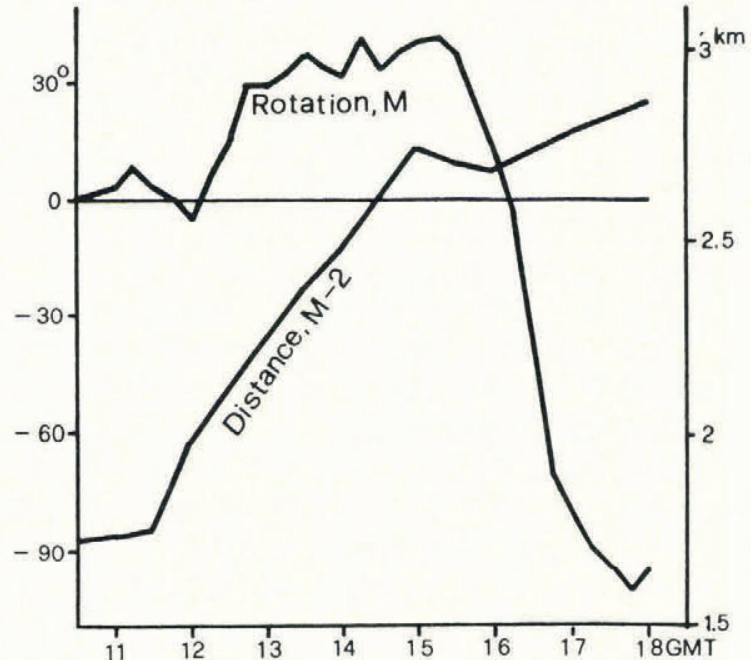

Fig.2. Rotation of the floe, on which the main station (M) was located. The orientation at 1030 GMT is taken as a reference $\left(0^{\circ}\right)$ and the clockwise rotation is shown with positive angle. The distance between stations $M$ and 2 (see Fig.1.) is also shown.

only, hence rotation components with higher frequencies are not presented. Other stations must have rotated in the same order of magnitude, but in a different way. Without the devices developed for the experiment, the measurements would have been impossible, as the reflector was rotated into an unfavourable direction most of the time. All of the three types satisfied the expectation, so that the distances were measured as shown in Fig.2 (the distance was measured with estimated accuracy of $\pm 2 \mathrm{~cm}$; the scale of the figure does not enable this to be shown).

Comments may be given on small points, so that an adequate system can be chosen for a particular purpose in future.

Type A: The frozen sea ice is not a flat surface. Ice ridges of various heights up to $5 \mathrm{~m}$ or more are present and disturb the measurements, by hiding the auxiliary station behind them. Although the reflector was raised to $2.5 \mathrm{~m}$ above sea level, the auxiliary station was not always visible from the main station. The measurement had to be made a the moment when the reflector was opportunely in sight. This was not so easy with type $\mathrm{A}$, as the time during which the reflector faced the observer was fixed and the reflector was not necessarily in sight during this time. On the other hand, the simple operation, without a remote control procedure, was confirmed to be a real advantage of this type.

Type B and C: The image of the auxiliary station was moving, not only horizontally, but also vertically, in the telescope of the theodolite and quite rapidly, as the ice floe of the observer was moving, rotating, and tilting, in addition to the movement of the auxiliary stations. Two screws of the theodolite had to be continuously operated, to keep the auxiliary station within the range of the telescope. At the same time, the remote control had to be managed. Everything had to be conducted rapidly, before the auxiliary station disappeared behind an ice edge. Two hands were simply not enough. One of the findings throughout the experiment was the fact that the measurement can be interrupted. When the theodolite did not follow the movement of the auxiliary station rapidly enough, or when a relatively small ice ridge passed in front of the reflector, the distance measurement was stopped and was reactivated as soon as the reflector was caught again. An accumulation of short time segments seemed to be sufficient for a measurement. The idea of another type was born from this: the mechanism is formally the same as that of type A, but with a much higher rotation rate, few/tens/hundreds of rotations per minute. The reflector faces the observer (in fact, any direction) at small time intervals. For those distance-measuring instruments which can function with 
numerous, chopped signals, instead of a single, continuous one, this type is attractive. Furthermore, a strictly simultaneous measurement of a marker, from more than one station, is possible.

\section{CONCLUSION}

Although the experiment was made on the pack ice, the device developed can be used generally under any circumstances, particularly on those (parts of) glaciers, which are moving rapidly, e.g. ice fall, calving, during surge. For this particular experiment, the reflector did not rotate around the horizontal axis, as both main and auxiliary stations remained at the sea surface. A generalization may be necessary for the glacier survey, by attaching an additional similar mechanism for the rotation around the horizontal axis or employing a cardan support.

\section{ACKNOWLEDGEMENT}

The Department of Geography, ETH Zürich (Head, Dr D. Steiner) supported the second and the third authors during the development and construction of the instrument. The crew of FS Polarstern (Captain, D. Zapff) and the scientists on board (chief scientist, Dr. E. Augstein) assisted the first author during the experiment, beyond their duty. 